\title{
Water Mine Model Recognition by Immune Neural Network
}

\author{
Haibo Liu, Jing Shen, and Guochang Gu \\ College of Computer Science and Technology, Harbin Engineering University, \\ Harbin 150001, China \\ \{liuhaibo, shenjing, guguochang\}@hrbeu.edu.cn
}

\begin{abstract}
An immune neural network (INN) is proposed for water mine model recognition. The INN is a two-layer Boolean network whose number of outputs is adaptive according to the task and the affinity threshold. The precision of the recognition results can be controlled through adjusting the affinity threshold. The INN has favorable capability of noise tolerance.
\end{abstract}

Keywords: water mine model, recognition, immune neural network.

\section{Introduction}

It is necessary for mine countermeasure systems to recognize the model of a water mine. Most of the previous researches on mine countermeasures [1][2][3] focused main attentions on the detection and classification of water mines. But the previous approaches can only classify the detected object as two classes, i.e., mine-like or not. In this paper, an immune neural network (INN) is constructed based on a modified clonal selection operation for water mine model recognition.

\section{Immune Neural Network}

Represent the feature data of water mines as an antigen population $A^{g}=\left\{A^{g}{ }_{1}, A^{g}{ }_{2} \ldots\right.$ $\left.A^{g}{ }_{n}\right\}$. An antigen can be represented as $l$-length bit string. Each bit represents a feature. The antibody repertoire, $A^{b}=\left\{A^{b}{ }_{1}, A^{b}{ }_{2}, \ldots, A^{b}{ }_{m}\right\}$, will be modeled as a Boolean competitive network with $l$ inputs, called INN. Employ an $l$-dimensional vector $w_{j}$ represents the weights connecting the inputs to the output unit $j$. Define the affinity $A_{i j}$ as the Hamming distance between $A^{g}{ }_{i}$ and $A^{b}$. For each $A^{g}{ }_{i}, v_{i}$ indexing the highest affinity antibody. The antigens concentration $c_{j}$ for $A_{j}^{b}$ can be calculated according to Eq.(1),

$$
c_{j}=\sum_{i=1}^{n} \operatorname{iif}\left(v_{i}=j, 1,0\right),
$$

where, $\operatorname{iif}\left(v_{i}=j, 1,0\right)$ means that if $v_{i}=j$ holds then return 1 else 0 . Define $e$ as an affinity threshold. For $A^{g}{ }_{i}$ and $A^{b}{ }_{j}$, if $l-A_{i j}>e$ then we can say that $A^{g}{ }_{i}$ and $A^{b}{ }_{j}$ are 
matched. Define $m_{j}$ as an affinity maturation indicator. If $m_{j}$ is True then stop cloning antibody $A^{b}$. For antibody $A_{j}^{b}$ and arbitrary $i \in\left\{i \mid v_{i}=j\right\}$, if $l-A_{i j}>e$ holds, then set $m_{j}$ = True as Eq.(2),

$$
m_{j}=\bigwedge_{i \in\left\{i \mid v_{i}=j\right\}}\left(l-A_{i j}>e\right)
$$

We developed a modified clonal selection algorithm for constructing an INN:

1) Initiate an INN with $n$ antigens $A^{g}{ }_{1}, A^{g}{ }_{2} \ldots A^{g}{ }_{n}$ and 1 antibody $A^{b}{ }_{1}$. For each integer $i \in[1, n]$, set $v_{i}=1$. Set each bit of $w_{i}$ to 1 . Set $c_{1}=n, m_{1}=$ False. Set $e$ to an appropriate value.

2) Determine the candidate $A^{b}{ }_{j}$ to be cloned according to Eq.(3). If $j$ is null then stop else continue step 3 ) to clone $A_{j}^{b}$.

$$
j=\arg \max _{j \in O} c_{j}, \text { where } O=\left\{j \mid c_{j}>1 \wedge m_{j}=\text { False }\right\}
$$

3) Select the worst matching $A^{g}{ }_{i}$ of $A^{b}{ }_{j}$ according to Eq.(4),

$$
i=\arg \min _{i \in\left\{i \mid v_{i}=j\right\}} A_{i j} .
$$

4) Clone a new antibody $A^{b}{ }_{k}$ with the weights $w_{k}$ of its antibody are the exact complement of $A^{g}$, then add $A^{b}{ }_{k}$ to INN.

5) For each antigen $A^{g} i$, update $v_{i}$. For each antibody $A_{j}^{b}$, update $c_{j}$ and $m_{j}$.

6) If all antibodies are mature then delete the antibodies with $c=0$ and stop, else goto step 2).

When the algorithm (MCSA for short) stops, an INN is constructed, each output of which represents one or several antigen patterns, i.e., water mine models. The number of patterns that an output represents is determined by the affinity threshold $e$. When $e$ $=0$, one output corresponds to one pattern. The bigger the value of $e$ is, the more patterns an output represents along with the lower precision.

The process of recognition is very simple. Given an antigen $A^{g}{ }_{i}$ as input, the INN computes the antibody being activated according to Eq.(5), then the water mine model can be recognized.

$$
j=\arg \max _{j \in O} A_{i j}, \quad \text { where } O=\left\{j \mid l-A_{i j}>e\right\}
$$

There is no output can be activated if $O=\Phi$, which means that $A^{g}{ }_{i}$ cannot be recognized, i.e., the water mine is not any known model or may be it is not a water mine at all.

\section{Simulation Experiments}

For constructing an INN, a water mine data set with models and binary attributes was prepared as shown in Table 1 . We applied MCSA to the data set with setting $l=20$, $n=21$, and $e=0$, which means that the MCSA will create a network with antibodies highly specific for each input pattern (antigen). Thus, it would be expected that the 
final network was composed of 21 antibodies, but the resultant network contained only 18 antibodies. This is because that the models (e.g., NM103 and WSM210) mapped into the same node are described as the same attribute strings.

Table 1. Water mine data set with their models and binary attributes

\begin{tabular}{|c|c|c|c|c|c|c|c|c|c|c|c|c|c|c|c|c|c|c|c|c|c|}
\hline & 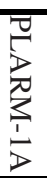 & 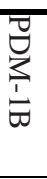 & 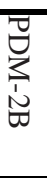 & $\begin{array}{l}\sqrt[3]{8} \\
\frac{3}{3} \\
3 \\
3\end{array}$ & 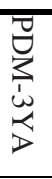 & & 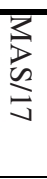 & 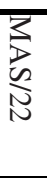 & 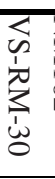 & & & & & & & & & & & & $\begin{array}{l}\overline{3} \\
\overline{3} \\
3 \\
3\end{array}$ \\
\hline \multicolumn{22}{|l|}{ Shape } \\
\hline hen & 0 & 0 & 0 & 1 & 0 & 1 & 0 & 1 & 0 & 0 & 0 & 0 & 0 & 0 & 0 & 0 & 0 & 0 & 0 & 0 & 0 \\
\hline cylin & 0 & 0 & 1 & 0 & 0 & 0 & 0 & 0 & 0 & 1 & 0 & 1 & 0 & 1 & 1 & 0 & 0 & 1 & 1 & 1 & 1 \\
\hline d-cone & 0 & 1 & 0 & 0 & 0 & 0 & 0 & 0 & 0 & 0 & 1 & 0 & 1 & 0 & 0 & 1 & 0 & 0 & 0 & 0 & 0 \\
\hline obla & 0 & 0 & 0 & 0 & 0 & 0 & 0 & 0 & 1 & 0 & 0 & 0 & 0 & 0 & 0 & 0 & 1 & 0 & 0 & 0 & 0 \\
\hline clut & 1 & 0 & 0 & 0 & 1 & 0 & 1 & 0 & 0 & 0 & 0 & 0 & 0 & 0 & 0 & 0 & 0 & 0 & 0 & 0 & 0 \\
\hline \multicolumn{22}{|c|}{ 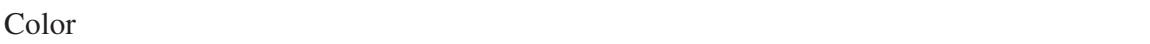 } \\
\hline green & 1 & 0 & 0 & 1 & 0 & 0 & 0 & 0 & 0 & 0 & 0 & 0 & 0 & 0 & 0 & 0 & 0 & 0 & 0 & 0 & 0 \\
\hline black & 0 & 0 & 1 & 0 & 0 & 0 & 0 & 0 & 0 & 0 & 0 & 0 & 0 & 0 & 0 & 0 & 0 & 0 & 0 & 0 & 1 \\
\hline gray & 0 & 0 & 0 & 0 & 0 & 0 & 0 & 1 & 1 & 1 & 1 & 0 & 0 & 1 & 1 & 1 & 1 & 1 & 1 & 1 & 0 \\
\hline re & 0 & 0 & 0 & 0 & 1 & 0 & 0 & 0 & 0 & 0 & 0 & 0 & 0 & 0 & 0 & 0 & 0 & 0 & 0 & 0 & 0 \\
\hline O1 & 0 & 0 & 0 & 0 & 0 & 0 & 0 & 0 & 0 & 0 & 0 & 1 & 1 & 0 & 0 & 0 & 0 & 0 & 0 & 0 & 0 \\
\hline ye & 0 & 1 & 0 & 0 & 0 & 0 & 1 & 0 & 0 & 0 & 0 & 0 & 0 & 0 & 0 & 0 & 0 & 0 & 0 & 0 & 0 \\
\hline brown & 0 & 0 & 0 & 0 & 0 & 1 & 0 & 0 & 0 & 0 & 0 & 0 & 0 & 0 & 0 & 0 & 0 & 0 & 0 & 0 & 0 \\
\hline \multicolumn{22}{|c|}{ 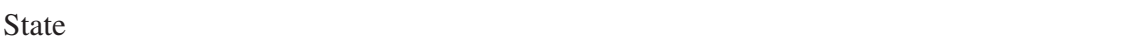 } \\
\hline floatir & 1 & 0 & 0 & 0 & 0 & 0 & 0 & 0 & 0 & 0 & 0 & 0 & 0 & 0 & 0 & 0 & 0 & 0 & 0 & 0 & 0 \\
\hline moo & 0 & 0 & 1 & 1 & 1 & 1 & 1 & 0 & 0 & 0 & 0 & 0 & 0 & 0 & 0 & 0 & 0 & 1 & 0 & 0 & 1 \\
\hline bott & 0 & 1 & 0 & 0 & 0 & 0 & 0 & 1 & 1 & 0 & 1 & 0 & 1 & 0 & 1 & 1 & 0 & 0 & 1 & 1 & 0 \\
\hline & 0 & 0 & 0 & 0 & 0 & 0 & 0 & 0 & 0 & 1 & 0 & 1 & 0 & 1 & 0 & 0 & 0 & 0 & 0 & 0 & 0 \\
\hline adsorpti & 0 & 0 & 0 & 0 & 0 & 0 & 0 & 0 & 0 & 0 & 0 & 0 & 0 & 0 & 0 & 0 & 1 & 0 & 0 & 0 & 0 \\
\hline \multicolumn{22}{|l|}{ Depth } \\
\hline deep & 0 & 0 & 0 & 0 & 0 & 0 & 0 & 0 & 0 & 1 & 1 & 1 & 1 & 1 & 1 & 1 & 0 & 1 & 1 & 1 & 1 \\
\hline shallov & 1 & 1 & 1 & 1 & 1 & 1 & 1 & 1 & 1 & 0 & 0 & 0 & 0 & 0 & 0 & 0 & 1 & 0 & 0 & 0 & 0 \\
\hline \multicolumn{22}{|l|}{ Has } \\
\hline antennae & 1 & 1 & 1 & 1 & 1 & 1 & 1 & 1 & 0 & 0 & 0 & 0 & 0 & 1 & 0 & 0 & 0 & 0 & 0 & 0 & 0 \\
\hline
\end{tabular}

In most cases, the sensor data may be incomplete or inaccurate. The INN can easily deal with these cases by adjusting the value of the affinity threshold $e$ when recognizes, which can be demonstrated by the following experiments. Random noise was inserted into the learning samples in Table 1 by simply reverting a bit 0 into a 1 , or vice-versa. We tested eleven different noise levels from $0 \%$ to $50 \%$, corresponding to the shift of 0 to 10 bits, respectively. The INN was tested for different values of the affinity threshold $e$ from 0 to 10 , and a correct recognition (CR) is assumed when the network maps the corrupted pattern into the same node as the original pattern. Fig.1 depicts the experiment results that are the average taken over ten runs. Fig.1 shows that the CR is affected both by the number of noise bits and the affinity threshold $e$. Keeping a value of $e$ constant, the CR decreases almost linearly with the noise level increasing. If we keep a constant noise level, the CR increases nonlinearly, i.e., the $\mathrm{CR}$ almost keeps constant when the value of $e$ is bigger than the number of noise bits. 
In these experiments, we did not take into account the effect of adjusting the affinity threshold $e$ when the INN is constructed. Apparently, the value of $e$ in recognition should be equal to or bigger than that in construction. It is an acceptable strategy to set $e$ in recognition to a bigger value when the noise level is uncertain.

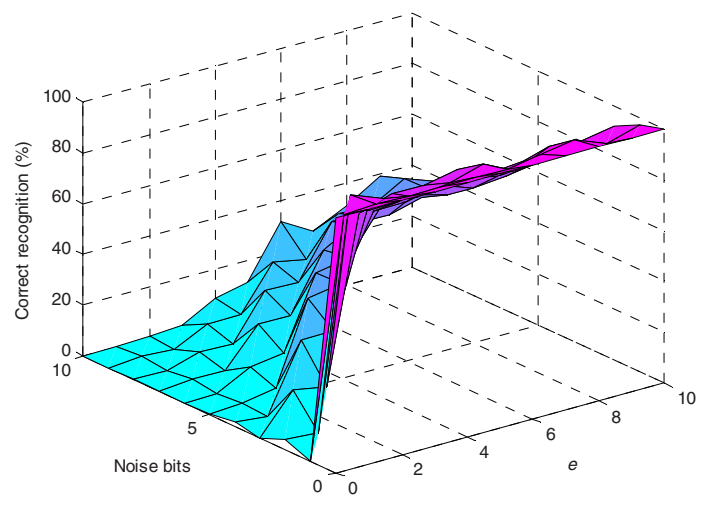

Fig. 1. The correct recognition rate of INN

\section{Conclusions}

The INN is a two-layer Boolean network whose number of outputs is adaptive according to the task and the affinity threshold, which is significant for solving machinelearning problems, such as knowledge acquisition and classification. The precision of recognition result can be controlled through adjusting the affinity threshold, which endows INN with satisfied noise tolerance capability. In the future, we will apply the INN to underwater minesweepers.

Acknowledgments. Supported by the Young Researchers Foundation of Heilongjiang under grant QC06C022, the Fundamental Research Foundation of Harbin Engineering University under grant HEUFT05021, HEUFT05068 and HEUFT07022.

\section{References}

1. Tuzlukov, V.P.: Probability of detection of mines and minelike targets in deep water using generalized detector with digital threshold device. Proc. of Detection and Remediation Technologies for Mines and Minelike Targets VI, Orlando, US (2001) 1283-1294

2. Aridgides, T., Fernandez, M., Dobeck G.J.: Improved processing string fusion approach investigation for automated sea mine classification in shallow water. Proc. of Detection and Remediation Technologies for Mines and Minelike Targets IX, Orlando, US (2004) 315-326

3. Ciany, C., Zurawski, W.: Improvements in computer aided detection/computer aided classification (CAD/CAC) of bottom mines through post analysis of a diverse set of very shallow water (VSW) environmental test data. Proc. of Detection and Remediation Technologies for Mines and Minelike Targets IX, Orlando, US (2004) 327-335 